\title{
Conocimiento y estigma sobre personas con esquizofrenia en estudiantes de enfermería
}

\section{Knowledge and stigma about people with schizophrenia in nursing students}

\author{
Itzel J. Pérez-Delgado ${ }^{a}$, Pedro U. González-Cruz ${ }^{b}$, María M. Camacho-Rodríguez ${ }^{c}$, Gustavo \\ Vélez-Díaz ${ }^{d}$ Edith A. Cano-Estrada ${ }^{e}$
}

\begin{abstract}
:
Background: Schizophrenia is a public health issue, which is displayed by behaviours outside social norms, lack of knowledge could influence on quality of nursing healthcare. People with this disorder are usually discriminated and stigmatized. Objective: Assess the knowledge and stigma among nursing students about people with schizophrenia. Material and methods: A Quantitative, descriptive, cross-sectional study design was applied. Non-probabilistic sampling $n=28$ students on sixth, seventh and eighth semester of the Bachelor's Degree in Nursing of The Escuela Superior of Tlahuelilpan; in March-May period 2021. The Perceived Devaluation and Discrimination toward mental illness Scale (PDDs) (a-Cronbach: 0.76) and The Knowledge about Schizophrenia Questionnaire (KASQ) were applied. ( $\alpha$-Cronbach: 0.72). Outcomes: (39.3\%) belongs to sixth semester students, $(25 \%)$ to those seventh ones and $35.7 \%$ to those eighth ones, (85.7\%9) are female and (14.3\%) male. (21.4\%) shows students with enough knowledge about Schizophrenia, meanwhile (78.6\%) shows students with lack of knowledge, $(3.6 \%)$ of these students shows low stigma (96.4\%) of these ones shows a high stigma. Conclusions: The knowledge about people with schizophrenia of the students of the Nursing Degree is deficient. Besides that, they show stigma.
\end{abstract}

\section{Keywords:}

Nursing Students, Social Stigma, Knowledge, Schizophrenia.

\section{Resumen:}

Introducción: La esquizofrenia es un problema de salud pública manifestado por conductas fuera de las normas sociales, la falta de conocimientos podría influir en la calidad de la atención de enfermería. Las personas que padecen este trastorno suelen ser discriminados y estigmatizados. Objetivo: Valorar el nivel de conocimiento y la presencia de estigma sobre las personas con esquizofrenia en los estudiantes de Enfermería. Material y métodos: Estudio cuantitativo, descriptivo, transversal, muestra no probabilística $n=28$ estudiantes de sexto, séptimo y octavo semestre de la Licenciatura en Enfermería de la Escuela Superior de Tlahuelilpan; en el periodo de marzo-mayo 2021. Se aplicó la Escala de devaluación y discriminación percibidas ( $\alpha$-Cronbach: 0.76) y el Cuestionario de conocimientos sobre la esquizofrenia ( $\alpha$-Cronbach: 0.72 ). Resultados: El 39.3\% de estudiantes corresponde a sexto semestre, $25 \%$ a séptimo y $35.7 \%$ a octavo, de ellos $85.7 \%$ son mujeres y $14.3 \%$ hombres. El $21.4 \%$ de los estudiantes de la Licenciatura en Enfermería de la Escuela Superior de Tlahuelilpan presenta un conocimiento suficiente mientras que el 78.6\% representa a estudiantes con un conocimiento deficiente, el $3.6 \%$ no presenta estigma y $96.4 \%$ presenta estigma. Conclusiones: Los conocimientos sobre personas con esquizofrenia de los estudiantes de la Licenciatura en Enfermería son deficientes y presentan estigma.

\section{Palabras Clave:}

Estudiantes de Enfermería, Estigma Social, Conocimiento, Esquizofrenia.

\footnotetext{
Autor de Correspondencia, Universidad Autónoma del Estado de Hidalgo, https://orcid.org/0000-0003-2528-9093, Email: pe397563@uaeh.edu.mx

${ }^{b}$ Universidad Autónoma del Estado de Hidalgo, https://orcid.org/0000-0002-4577-2624, Email: go404730@uaeh.edu.mx

${ }^{c}$ Universidad Autónoma del Estado de Hidalgo, https://orcid.org/0000-0002-2897-0354, Email: ca323838@uaeh.edu.mx

${ }^{d}$ Universidad Autónoma del Estado de Hidalgo, https://orcid.org/0000-0002-2680-1944, Email: gustavo_velez@uaeh.edu.mx

${ }^{e}$ Universidad Autónoma del Estado de Hidalgo, https://orcid.org/0000-0002-8315-1087, Email: edith_cano@uaeh.edu.mx
} 


\section{Introducción}

La esquizofrenia es un trastorno mental grave que afecta a casi 21 millones de personas en todo el mundo. Según la Organización Mundial de la Salud (OMS) es un trastorno caracterizado por una distorsión del pensamiento, las percepciones, las emociones, el lenguaje, la conciencia de sí mismo y la conducta [1]. En el cerebro existen miles de millones de neuronas y cada una de ellas tiene ramificaciones o terminales que transmiten y reciben información de otras neuronas. Estas terminales producen sustancias químicas llamadas neurotransmisores, que llevan los mensajes de la punta de una terminal hasta la punta de otra. En el cerebro de los pacientes que tienen esquizofrenia existe una alteración en la comunicación entre las neuronas. Por otro lado, se considera que la esquizofrenia es causada por un trastorno orgánico, en el cerebro existe una sustancia llamada dopamina, esta sustancia se produce en exceso y da por resultado los síntomas que caracterizan esta enfermedad. Sin embargo, existen otros factores para padecer esquizofrenia como lo son los hereditarios [2].

La esquizofrenia se divide en diferentes categorías y es por esto que tener la información correcta y no dejarse llevar por la mal información permitirá que se atienda de forma correcta a los pacientes y que se pueda identificar los diferentes tipos de episodios y estados crónicos que llegan a presentarse.

Las personas esquizofrénicas suelen sufrir estigmatización, discriminación y la violación de sus derechos humanos [1]. En México más de un millón de personas padece esquizofrenia [3] y a menudo son considerados agresivos, peligrosos e incapaces de trabajar, sin embargo, al recibir un tratamiento se mantendrán estables.

Los estudiantes de enfermería son considerados principiantes [4] y van adquiriendo el conocimiento por medio de la experiencia práctica, sin embargo, el conocimiento teórico es importante ya que se complementará con el conocimiento práctico, el estudiante de enfermería debe saber qué hacer y por qué lo hace.

En México existen 15 enfermeras por cada mil habitantes, pero no todas las personas que alcanzan el grado de enfermero ejercen su profesión, esto se refleja en las estadísticas internacionales, donde México tiene 2.9 enfermeros por cada mil habitantes [5]. Los graduados en enfermería deberían tener una predisposición diferente ante la enfermedad mental, esto generaría una reducción del estigma de forma que participarían del fomento por la aceptación, la integración y la igualdad de las personas afectadas por esquizofrenia.

Al ser los profesionales de enfermería el contacto directo con los pacientes en su tratamiento y también aquellos que brindan atención y cuidados, deben contar con las mejores prácticas de cuidado basadas en el conocimiento teórico y práctico.

El objetivo de la investigación fue valorar el conocimiento y la presencia de estigma sobre personas con esquizofrenia en estudiantes de enfermería. Se estimó, identificó y clasificó el nivel de conocimiento y estigma de los alumnos de Enfermería sobre personas con esquizofrenia.

\section{Material y Métodos}

Estudio cuantitativo, descriptivo, prolectivo, transversal, de muestra no probabilística. La población estudiada fueron estudiantes de enfermería de la Escuela Superior de Tlahuelilpan, se tuvo un total de 28 estudiantes que pertenecían a sexto, séptimo y octavo semestre. Se tomaron en cuenta criterios de inclusión que los participantes debían cumplir como estar inscritos en la Universidad Autónoma del Estado de Hidalgo, Escuela Superior de Tlahuelilpan y estar cursando el sexto, séptimo u octavo semestre al momento de ser aplicados los instrumentos.

Una vez que se identificaron a los estudiantes, fueron contactados a través de WhatsApp por medio de su jefe de grupo y tutor, se les envió la invitación para participar en la investigación, los estudiantes que voluntariamente se ofrecieron a participar tuvieron acceso al enlace del Formulario de Google. Dentro del formulario se especificó que toda la información que proporcionaran sería registrada de manera anónima y sería de uso exclusivo para la investigación, al estar de acuerdo, los estudiantes podían proseguir a llenar el formulario empezando por las variables sociodemográficas y posteriormente los instrumentos. Para asegurar que sólo estudiantes de la Escuela Superior de Tlahuelilpan contestaran el formulario, se restringió el acceso a cuentas con correo institucional. Todos los datos se recabaron en el periodo de marzo-mayo de 2021.

Se usaron dos instrumentos, el primero fue el Cuestionario de conocimientos sobre la esquizofrenia que consta de 9 ítems en escala de Likert. Este cuestionario cuenta con una consistencia interna global de 0.72 de la prueba estadística de Alfa de Cronbach. El segundo instrumento fue la Escala de Devaluación y Discriminación Percibidas (DDS) que tiene dos dimensiones con 19 ítems en escala de Likert. Dicho instrumento cuenta con una consistencia interna global de 0.76 , obtenida a través de la prueba 
estadística de Alfa de Cronbach. También se realizó un cuestionario con las variables sociodemográficas de relevancia para la investigación tales como edad, semestre que cursaban, género, estado civil, carrera técnica previa y promedio.

El análisis se realizó con Statistical Package for Social Sciences (SPSS) v.25 en el cual se hizo la estadística descriptiva de las variables sociodemográficas obteniendo frecuencias y porcentajes por cada variable, así como para las variables de estudio. Cuando se obtuvieron las respuestas de los participantes, se registraron en una base de datos en Excel y posteriormente se exportaron al SPSS.

Para llevar a cabo esta investigación se redactó un documento en donde se solicitaba la autorización para aplicar los instrumentos a los estudiantes de enfermería, este documento fue dirigido a la directora de la Escuela Superior de Tlahuelilpan.

Toda la información recabada fue de uso exclusivo para el estudio, los datos de cada estudiante permanecen de manera anónima.

\section{Resultados}

Se registró la información de 28 estudiantes de los cuales, $39.3 \%$ son de sexto semestre $(n=11), 25 \%$ a séptimo semestre $(n=7)$ y $50 \%$ a octavo $(n=10)$. El $85.7 \%$ fueron mujeres $(n=24)$ y el $14.3 \%$ hombres $(n=4)$. El $32.1 \%$ cuenta con estudios técnicos de enfermería $(n=9)$ y el $67.9 \%$ no cuenta con estudios técnicos $(n=19)$.

El cuestionario de conocimientos sobre la esquizofrenia registró que sólo el $21.4 \%$ de la muestra obtuvo la puntuación esperada, con esto se puede estimar que el conocimiento de los estudiantes es suficiente mientras que $78.6 \%$ tiene conocimiento deficiente.

El $90.9 \%$ de los estudiantes cree que las personas con esquizofrenia oyen voces, $66.7 \%$ considera que estos pacientes tienen personalidad dividida 0 múltiple, el $15.2 \%$ no lo sabe y el $18.2 \%$ considera que no es así. El $84.8 \%$ cree que las personas con esquizofrenia presentan tendencia al aislamiento social y el $15.2 \%$ opina que no. El $75.8 \%$ considera que estos pacientes presentan falta de voluntad mientras que el resto no lo sabe. El $48.4 \%$ cree que las personas con esquizofrenia padecen deficiencia mental, el $36.4 \%$ cree que no y el $15.2 \%$ no lo sabe. El $87.9 \%$ considera que las personas con esquizofrenia presentan conductas agresivas y que son peligrosas mientras que el $12.1 \%$ cree lo contrario. El $87.9 \%$ considera que estos pacientes presentan conductas raras o inadecuadas y el resto no lo sabe. El $57.6 \%$ sabe que los pacientes que padecen esquizofrenia no tienen cura y el $42.4 \%$ cree que sí. El $87.9 \%$ considera que el tratamiento les permite a los pacientes mantenerse estables y el $12.1 \%$ opina lo contrario.

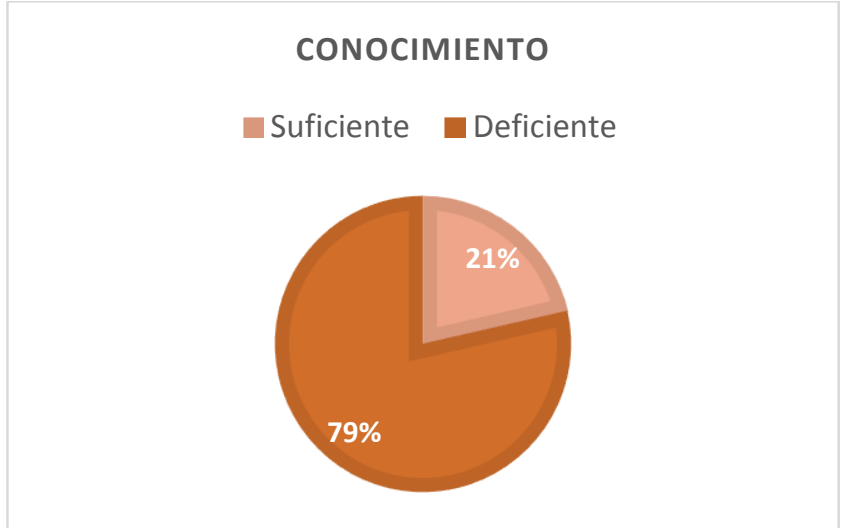

Gráfico 1. Resultados en porcentaje sobre el conocimiento de esquizofrenia.

(Fuente: Elaboración propia).

De acuerdo con los datos obtenidos en el instrumento Escala de Devaluación y Discriminación Percibidas (DSS), sólo el $3.6 \%$ de los estudiantes obtuvo la puntuación esperada, por esto se estima que no presentaban estigma y el $96.4 \%$ de la muestra sí presentó estigma.

\section{ESTIGMA}

Presenta $\square$ No presenta

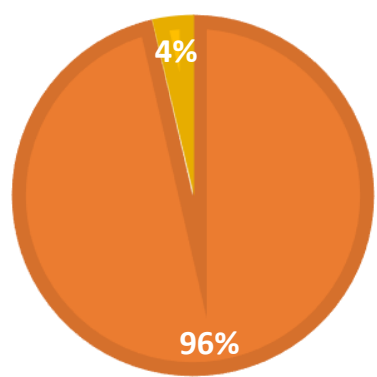

Gráfico 2. Resultados en porcentaje sobre el estigma hacia las personas con esquizofrenia.

(Fuente: Elaboración propia).

El $67.8 \%$ de los estudiantes aceptaría como amigo a una persona que tuvo una enfermedad mental severa como la esquizofrenia y el $32.2 \%$ respondió negativamente. El $42.9 \%$ cree que un paciente mental es tan inteligente como cualquier otro, mientras que el $57.1 \%$ cree lo contrario. El $28.6 \%$ considera que una persona con esquizofrenia es tan confiable como cualquier otra persona y el $71.4 \%$ considera que no son personas confiables. El $39.2 \%$ cree que ingresar a un servicio psiquiátrico representa un fracaso personal y el $60.8 \%$ respondió positivamente. El $87.9 \%$ considera que las personas con esquizofrenia son menospreciadas mientras que el $10.7 \%$ cree que no lo son. El $57.1 \%$ de 
los estudiantes cree que los pacientes esquizofrénicos son violentos mientras que el $42.9 \%$ considera que no lo son. El $32.2 \%$ no sentiría miedo al estar frente a un paciente esquizofrénico mientras que el $67.8 \%$ sí. El $50 \%$ de los estudiantes se avergonzaría de los pacientes con esquizofrenia mientras que el otro $50 \%$ no lo haría. El $32.1 \%$ de los estudiantes creen que las personas que tienen esquizofrenia están pagando por algo que hicieron y el $67.9 \%$ opina lo contrario.

\section{Discusión}

Los datos obtenidos nos permiten percibir que los estudiantes de enfermería, ante un trastorno mental como lo es la esquizofrenia, carecen de conocimientos suficientes llevando a su estigmatización. Los instrumentos utilizados nos indican que muy pocos estudiantes tienen un conocimiento satisfactorio ante este trastorno, por lo tanto, el estigma es predominante, por lo que es necesario reforzar los conocimientos sobre este tema en el programa educativo donde se ofrece la materia de salud mental. El $85.7 \%$ fueron mujeres porcentaje parecido al estudio realizado en Barcelona [6], ya que en esta carrera predomina el sexo femenino. Se han realizado estudios sobre la actitud de los estudiantes hacia las personas con demencia; sin embargo, en el presente estudio se obtuvieron porcentajes más altos en algunas opiniones, por ejemplo, el $32.1 \%$ de los estudiantes cree que las personas con esquizofrenia están pagando por algo que hicieron, porcentaje superior al obtenido en un estudio realizado en la Escuela Universitaria de Enfermería de San Joan de Déu [6]. Por otra parte, el $66.7 \%$ está convencido que estos pacientes presentan un problema de personalidad dividida 0 múltiple, porcentaje superior al que se obtuvo en el estudio de la Escuela Universitaria de Enfermería de San Joan de Déu y a otro estudio realizado en Chile [6] [7]. Además, el $28.6 \%$ considera que los pacientes esquizofrénicos son débiles de carácter, resultado superior al obtenido en el estudio realizado en la ciudad de Barcelona [6]. En contraste se encontraron algunas otras opiniones donde el porcentaje obtenido en el presente estudio resulto bajo en comparación con otros estudios, tales como, el $12.1 \%$ considera que la esquizofrenia tiene cura, porcentaje bajo con relación a los estudios antes mencionados [6] [7]. Cabe destacar que el $87.9 \%$ de los estudiantes de enfermería del presente estudio cree que las personas que sufren esquizofrenia son agresivas mientras que el estudio realizado en Barcelona sólo 44.3\% cree esto [6]. Incluso, el $53.6 \%$ consideró que no saldría con una persona esquizofrénica estable, porcentaje inferior al obtenido en el estudio de Barcelona [6]. Por lo tanto, la actitud de los estudiantes de enfermería en México, a diferencia de la actitud en otros países se inclinó a ser más negativa, teniendo mayores estigmas, posiblemente debido a la falta de conocimiento y acercamiento con las personas que sufren este tipo de demencia. Cabe señalar que el estudio tiene la limitación de la muestra que es pequeña por lo que se sugiere que en estudios posteriores poder ampliar la muestra e indagar en diferentes partes de la población ya que el aspecto sociocultural puede ser un factor determinante para formular un juicio hacia las personas con demencia, específicamente con esquizofrenia.

\section{Conclusión}

Los estudiantes de enfermería tienen conocimientos deficientes y presentan estigma sobre las personas con esquizofrenia. Es necesario buscar estrategias de aprendizaje que permita a los estudiantes adquirir el conocimiento que necesitan para que esto no influya en la calidad de su atención y cuidados. Además, es necesario indagar sobre los aspectos sociales y culturales que influyen para tener un estigma hacia las personas que sufren esquizofrenia.

\section{Referencias}

[1] OMS. Esquizofrenia [Internet]. Organización Mundial de la Salud; 2019 [consultado 2021 Oct 02]. Disponible en: https://www.who.int/es/news$\mathrm{room} /$ fact-sheets/detail/schizophrenia

[2] INNN. Esquizofrenia. [Internet]. México: INNN; 2017. [consultado 2021 Oct $\quad$ 02]. http://www.innn.salud.gob.mx/interna/medica/padecimientos/esquizofr enia.html

[3] Secretaría de Salud. Trastorno mental crónico y grave. [Internet]. México: Secretaría de Salud; 2017. [consultado 2021 Oct 02]. Disponible en: https://www.gob.mx/salud/articulos/en-mexico-mas-deun-millon-de-personas-padece-esquizofrenia

[4] Alligood M, Tomey A. Modelos y teorías en enfermería. 7th ed. España: ELSEVIER; 2011.

[5] OCDE. Código F. Actualización de Estadísticas de Salud de la OCDE 2019. [Internet]. México: Código F; 2019 Oct [consultado 2021Oct 03]. Disponible en: https://codigof.mx/estadisticas-en-salud-de-mexicoactualizacion-de-estadisticas-de-salud-de-la-ocde-2019/

[6] González, L. Prat, R., Morales, E., Artero, M., Ortega, A., Roldán, J. Estudio del Estigma de la Esquizofrenia en los Estudiantes de Enfermería. AEESME. 2009; 63 (1): p. 93-96.

[7] Grandón, P., Vielma-Aguilera, A., Bustos, C, Castro-Alzate, E., Saldivia, S. Evaluación del estigma hacia personas con diagnóstico de esquizofrenia mediante una escala de conocimiento. rev colomb psiquiat. 2018;47(2):72-81 